\title{
THE HAHN METRIC ON RIEMANN SURFACES
}

\author{
By C. DAvid Minda
}

\begin{abstract}
Recently, K. T. Hahn introduced a new pseudodifferential metric for complex manifolds by means of an extremal problem. This metric is investigated in the special case of Riemann surfaces. First, the metric is related to two other extremal problems on a Riemann surface. Next, basic properties of the Hahn metric are studied; in particular, it is shown that the Hahn metric is complete if it is nontrivial. For simply and doubly connected Riemann surfaces the Hahn metric is explicitly calculated; it is also studied on tori. Finally, the Hahn metric is shown to have generalized Gaussian curvature at least -4 ; for the unit disk it has constant curvature -4 .
\end{abstract}

\section{Introduction.}

K. T. Hahn introduced a new pseudo-differential metric and related distance function on complex manifolds via an extremal problem [4]. We shall investigate this metric in the context of Riemann surfaces. Let us begin by defining the metric. Let $\boldsymbol{B}=\{w:|w|<1\}$ denote the open unit disk. For an arbitrary Riemann surface $X$, let $\mathcal{S}(\boldsymbol{B}, X)$ denote the family of all injective analytic functions $f . \boldsymbol{B}$ $\rightarrow X$. Fix $p \in X$ and consider any local coordinate $z$ defined in a neighborhood of $p$ such that $z(p)=0$. Set

$$
S_{X}(z)=\inf \left\{\frac{1}{\left|(z \circ f)^{\prime}(0)\right|}: f \in \mathcal{S}(\boldsymbol{B}, X) \text { and } f(0)=p\right\} .
$$

A function $f \in \mathcal{S}(\boldsymbol{B}, X)$ is called extremal if $S_{X}(z)=1 /\left|(z \circ f)^{\prime}(0)\right|$ and $f(0)=p$. The quantity $S_{X}(z)$ depends upon the choice of the local coordinate $z$ at $p$ in such a way that $S_{X}(z)|d z|$ is an invariant form, or metric, on $X$. We shall call $S_{X}(z)|d z|$ the Hahn metric on $X$. Hahn [4] proved that $S_{X}(z)|d z|$ is a continuous metric on $X$. The Hahn metric naturally induces a distance function on $X$. For $p, q \in X$ set

$$
\mathcal{S}_{X}(p, q)=\inf \int_{r} S_{X}(z)|d z|,
$$

where the infimum is taken over all piecewise smooth paths $\gamma$ on $X$ that join $p$ and $q$.

Received February 24, 1982 
In section 2 we relate the Hahn metric to two other extremal problems on a Riemann surface. This will enable us to show that if the Riemann surface $X$ is not conformally equivalent to the complex plane $\boldsymbol{C}$ or the Riemann sphere $\boldsymbol{P}$, then the Hahn metric is positive at each point of $X$. Consequently, the distance function $S_{X}$ is compatible with the topology for such surfaces. The connection with these other extremal problems will also permit us to demonstrate the existence and essential uniqueness of extremal functions for the Hahn metric. Section 3 is devoted to several basic properties of the Hahn metric, including the facts that for hyperbolic Riemann surfaces it dominates the hyperbolic metric and is complete. Recall that a Riemann surface is called hyperbolic if the unit disk $\boldsymbol{B}$ is its universal covering surface. The Hahn metric for a torus and doubly connected Riemann surfaces is determined in sections 4 and 5 . Finally, curvature properties of the Hahn metric are discussed in section 6. In particular, we show that the Hahn metric has generalized Gaussian curvature at least -4 for any Riemann surface not conformally equivalent to either $\boldsymbol{C}$ or $\boldsymbol{P}$. For $\boldsymbol{B}$ the Hahn metric has constant curvature -4 .

\section{Connection with other extremal problems.}

In this section we relate the Hahn metric to two other extremal problems that involve the concepts of mapping radius and reduced modulus.

Let us start by recalling the definition of the mapping radius and formulating the extremal problem that involves the mapping radius. Assume $X$ is a Riemann surface. Let $\Omega$ be a hyperbolic simply connected region on $X$; that is, $\Omega$ is conformally equivalent to $\boldsymbol{B}$. Suppose $p \in \Omega$ and $z$ is a local coordinate defined in a neighborhood of $p$ such that $z(p)=0$. Then there is a unique conformal mapping $g$ of $\Omega$ onto a disk $\{w:|w|<r\}$ with $g(p)=0$ and the derivative of $g \circ z^{-1}$ has value 1 at the origin. The value of $r$ is uniquely determined by the choice of the local coordinate $z$. The value $r=r_{\Omega}(z)$ is called the mapping radius of $\Omega$ at $p$ with respect to the local coordinate $z$. The value of the mapping radius depends upon the choice of local coordinate at $p$ in such a way that $r_{\Omega}(z) /|d z|$ is an invariant expression [12]. Now, we can formulate the extremal problem. Define

$$
r_{X}(z)=\sup r_{\Omega}(z),
$$

where the supremum is taken over all hyperbolic simply connected regions on $X$ that contain $p$. The quantity $r_{X}(z)$ is called the mapping radius of $X$ at $p$ with respect to the local coordinate $z$. Clearly, the expression $r_{X}(z) /|d z|$ is invariant. A direct application of the Riemann mapping theorem shows that $S_{X}(z)|d z|=$ $|d z| / r_{X}(z)$.

Next, we recall the definition of the reduced modulus of a simply connected region on a Riemann surface. Suppose $X$ is a Riemann surface and $\Omega$ is a hyperbolic simply connected region on $X$. Assume $p \in \Omega$ and $z$ is a local coordinate in a neighborhood of $p$ such that $z(p)=0$. For all sufficiently small $r>0$ the 
closed disk $\overline{D(p, r)}=\{q \in X:|z(q)| \leqq r\}$ lies in $\Omega$. Then $\Omega(r)=\Omega \backslash \overline{D(p, r)}$ is a doubly connected region on $X$ and we denote its modulus by $M(r)$. Remember that if $\Omega(r)$ is conformally equivalent to an annulus $\left\{w: r_{1}<|w|<r_{2}\right\}$, then $M(r)=$ $\log \left(r_{2} / r_{1}\right)$. The sum $M(r)+\log r$ is increasing as $r$ tends to zero and the limit

$$
M_{\Omega}(z)=\lim _{r \rightarrow 0}(M(r)+\log r)
$$

is called the reduced modulus of $\Omega$ at $p$ with respect to the local coordinate $z$. The reduced modulus depends upon the choice of local coordinate at $p$ in such a way that $\exp \left(M_{\Omega}(z)\right) /|d z|$ is invariant and it is related to the mapping radius by

$$
r_{\Omega}(z)=\exp \left(M_{\Omega}(z)\right)
$$

[12]. The extremal problem for the reduced modulus is

$$
M_{X}(z)=\sup M_{\Omega}(z),
$$

where the supremum is taken over all hyperbolic simply connected regions $\Omega$ on $X$ with $p \in \Omega$. The quantity $M_{X}(z)$ is called the reduced modulus of $X$ at $p$ with respect to the local coordinate $z$. Clearly, $\exp \left(M_{X}(z)\right) /|d z|$ is invariant and

$$
S_{X}(z)|d z|=\frac{|d z|}{r_{X}(z)}=\exp \left(-M_{X}(z)\right)|d z| \text {. }
$$

The solution to the extremal problem for the reduced modulus is a very special case of a result of Jenkins [7]; the particular instance that we require is explicitly stated in [8]. We summarize it here. Assume that $X$ is a Riemann surface that is not conformally equivalent to either $\boldsymbol{C}$ or $\boldsymbol{P}$. As usual, $p \in X$ and $z$ is a local coordinate in a neighborhood of $p$ with $z(p)=0$. There is a unique hyperbolic simply connected region $\Omega^{*}$ on $X$ such that $p \in \Omega^{*}$ and $M_{\Omega^{*}}(z)$ is maximal for all hyperbolic simply connected regions on $X$ that contain $p$. The extremal region $\Omega^{*}$ is obtained by slitting $X$ along the trajectories of a quadratic differential $Q(w) d w^{2}$, holomorphic on $X$ apart from a double pole at $p$, with limiting end points at the zeros of $Q(w) d w^{2}$ together with possibly certain trajectories running from the ideal boundary of $X$ back to itself. $\Omega *$ is a circle domain in the trajectory structure of $Q(w) d w^{2}$. Note that the extremal region $\Omega^{*}$ does not depend upon the choice of the local coordinate at $p$. However, the value of the reduced modulus of $\Omega^{*}$ does clearly depend on the local coordinate, but the value is maximal with respect to any local coordinate. Thus,

$$
S_{X}(z)=\exp \left(-M_{\Omega^{*}}(z)\right) \text {. }
$$

In particular, if $X$ is a Riemann surface that is not conformally equivalent to either $\boldsymbol{C}$ or $\boldsymbol{P}$, then we can conclude that $S_{X}(z)|d z|$ is a positive metric on $X$. What is the situation if $X$ is conformally equivalent to $\boldsymbol{C}$ or $\boldsymbol{P}$ ? First, suppose $X=\boldsymbol{C}$ and $a \in \boldsymbol{C}$. For $R>0$ define $f_{R}(z)=a+R z$. Then $f_{R} \in \mathcal{S}(\boldsymbol{B}, \boldsymbol{C}), f(0)=a$ and $\left|f_{R}^{\prime}(0)\right|=R$. Hence, $S_{C}(a) \leqq 1 / R$, so $S_{C}(a)=0$. This gives $S_{C}(z)|d z|=0$. Similarly, 
$S_{\boldsymbol{P}}(z)|d z|=0$. In fact, if $X$ is conformally equivalent to either $\boldsymbol{C}$ or $\boldsymbol{P}$, then $S_{X}(z)|d z|=0$.

The result of Jenkins also enables us to show that extremal functions for the Hahn metric exist and are essentially unique if $X$ is not conformally equivalent to either $\boldsymbol{C}$ or $\boldsymbol{P}$. For $p \in X$ let $\Omega^{*}$ be the unique hyperbolic simply connected region containing $p$ that has maximal reduced modulus at $p$. Let $\phi: B \rightarrow$ $\Omega^{*}$ be a conformal mapping such that $\phi(0)=p$. From our discussion of the mapping radius and reduced modulus, we conclude that

$$
S_{X}(z)=\exp \left(-M_{\Omega^{*}}(z)\right)=\frac{1}{\left|(z \circ \phi)^{\prime}(0)\right|},
$$

where $z$ is a local coordinate at $p$ with $z(p)=0$. Thus, $\phi$ is an extremal function. Let us show that $\phi$ is unique up to precomposition with a rotation of $\boldsymbol{B}$. Suppose $f \in \mathcal{S}(\boldsymbol{B}, X)$ with $f(0)=p$ is also extremal. If $\Omega=f(\boldsymbol{B})$, then

$$
S_{X}(z)=\frac{1}{\left|(z \circ f)^{\prime}(0)\right|}=\exp \left(-M_{\Omega}(z)\right)
$$

Then $M_{\Omega}(z)=M_{\Omega^{*}}(z)$ and so Jenkins' result implies that $\Omega=\Omega^{*}$. Clearly, $\phi^{-1} \circ f(z)$ is a conformal automorphism of $\boldsymbol{B}$ that fixes the origin, so it is a rotation, say $e^{i \theta} z$. Then $f(z)=\phi\left(e^{i \theta} z\right)$.

\section{Basic properties of the Hahn metric.}

In this section we study some of the elementary fundamental properties of the Hahn metric. We begin by stating a result of Hahn [4] which shows that injective analytic functions are distance decreasing relative to the Hahn metric. Recall that if $f: X \rightarrow Y$ is an analytic function and $\rho(z)|d z|$ is a metric on $Y$, then $f^{*}(\rho(z)|d z|)$ denotes the pull-back to $X$ via $f$ of the metric $\rho(z)|d z|$. $f^{*}(\rho(z)|d z|)$ is a metric on $X$. If $X$ and $Y$ are plane regions, then $f^{*}(\rho(z)|d z|)$ $=\rho(f(z))\left|f^{\prime}(z)\right||d z|$.

Theorem 1. Suppose $X$ and $Y$ are Riemann surfaces and $f: X \rightarrow Y$ is an injective analytıc function. Then $f^{*}\left(S_{Y}(z)|d z|\right) \leqq S_{X}(z)|d z|$. In partıcular, if $f: X \rightarrow Y$ is a conformal mapping, then $f^{*}\left(S_{Y}(z)|d z|\right)=S_{X}(z)|d z|$.

Corollary 1. Let $X$ be a Riemann surface. Then $S_{X}(z)|d z|$ is invariant under the group Aut $(X)$ of conformal automorphism of $X$; that $\imath$, $f *\left(S_{X}(z)|d z|\right)$ $=S_{X}(z)|d z|$ for all $f \in \operatorname{Aut}(X)$.

COROLlARY 2. If $X$ and $Y$ are Riemann surfaces with $X \subset Y$, then $S_{X}(z)|d z|$ $\geqq S_{Y}(z)|d z|$.

Proof. Just apply Theorem 1 to the inclusion mapping of $X$ into $Y$.

Corollary 2 expresses a monotonicity property of the Hahn metric. Strict 
inequality need not hold in this result even if $X$ is a proper subsurface of $Y$. This can be seen as follows. Suppose $Y$ is a nonsimply connected Riemann surface and $p \in Y$. Let $\phi \in \mathcal{S}(\boldsymbol{B}, X)$ be an extremal function, so that $\phi(0)=p$ and $S_{Y}(z)=1 /\left|(z \circ \phi)^{\prime}(0)\right|$, where $z$ is any local coordinate at $p$ such that $z(p)=0$. Then $X=\phi(\boldsymbol{B})$ is a simply connected subsurface of $Y$ and $S_{X}(z)=1 /\left|(z \circ \phi)^{\prime}(0)\right|=$ $S_{Y}(z)$.

Next, we want to compare the Hahn metric to the hyperbolic metric. Let $X$ be a hyperbolic Riemann surface; that is, $\boldsymbol{B}$ is the universal covering surface of $X$. Suppose $\pi: B \rightarrow X$ is any analytic universal covering projection. The hyperbolic metric $\lambda_{X}(z)|d z|$ on $X$ is real-analytic and has constant (Gaussian) curvature -4 . It is the unique metric on $X$ that satisfies

$$
\pi^{*}\left(\lambda_{X}(z)|d z|\right)=\lambda_{B}(z)|d z|=\frac{|d z|}{1-|z|^{2}} .
$$

It is also the Kobayashi-Royden metric on $X$. That is, if $p \in X$ and $z$ is a local coordinate at $p$ with $z(p)=0$, then

$$
\lambda_{X}(z)=\inf \left\{\frac{1}{\left|(z \circ f)^{\prime}(0)\right|}: \quad f: B \longrightarrow X \text { is analytic and } f(0)=p\right\} .
$$

An analytic function $f: B \rightarrow X$ is extremal for the hyperbolic metric if and only if it is a universal covering that satisfies $f(0)=p$. Obviously, the Hahn metric dominates the hyperbolic metric; the following theorem sharpens this result.

THEOREM 2. Let $X$ be a hyperbolic Riemann surface. Then $\lambda_{X}(z)|d z| \leqq$ $S_{X}(z)|d z|$ and equality holds if and only if $X$ is simply connected.

Proof. Fix $p \in X$ and let $z$ be a local coordinate in a neighborhood of $p$ such that $z(p)=0$. Assume $\pi: \boldsymbol{B} \rightarrow X$ is an analytic universal covering with $\pi(0)=p$. Then

$$
\lambda_{X}(z)=\frac{1}{\left|(z \circ \pi)^{\prime}(0)\right|} .
$$

Let $\phi \in S(\boldsymbol{B}, X)$ be an extremal function for the Hahn metric at $p$; that is, $\phi(p)=0$ and

$$
S_{X}(z)=\frac{1}{\left|(z \circ \phi)^{\prime}(0)\right|}
$$

Since $\pi$ is a universal covering, there is a holomorphic function $h: B \rightarrow \boldsymbol{B}$ with $h(0)=0$ such that $\phi=\pi \circ h$. Then

$$
\lambda_{X}(z)=\left|h^{\prime}(0)\right| S_{X}(z) .
$$

Schwarz' Lemma implies that $\left|h^{\prime}(0)\right| \leqq 1$ with equality if and only if $h$ is a rotation of $\boldsymbol{B}$. Thus, $\lambda_{X}(z) \leqq S_{X}(z)$. If $X$ is simply connected, then $\pi: B \rightarrow X$ is a conformal mapping. This gives $h(w)=e^{i \theta} w$ for some $\theta \in \boldsymbol{R}$, so equality holds. 
Conversely, suppose that equality holds. Then $\left|h^{\prime}(0)\right|=1$ so $h(z)=e^{\imath \theta} w$, or $\phi(w)$ $=\pi\left(e^{\imath \theta} w\right)$. This shows that $\pi$ is injective, so $X$ must be simply connected.

Corollary. Let $X$ be a Riemann surface. The distance function $\mathcal{S}_{X}$ is complete if $X$ is not conformally equivalent to either $\boldsymbol{C}$ or $\boldsymbol{P}$.

Proof. First, we assume that $X$ is hyperbolic. Let $d_{X}$ denote the hyperbolic distance function on $X$ induced by the hyperbolic metric. Then $d_{X}$ is compatible with the topology of $X$ and complete [3, p. 204]. Theorem 2 implies that $d_{X} \leqq \mathcal{S}_{X}$. It follows easily that $\mathcal{S}_{X}$ is complete. If $X$ is neither hyperbolic nor conformally equivalent to $\boldsymbol{C}$ or $\boldsymbol{P}$, then $X$ is conformally equivalent to $\boldsymbol{C} \backslash\{0\}$ or a torus. The explicit formula for $S_{C \backslash \text { iol }}(z)|d z|$ given in section 5 implies that $S_{C \backslash 0\}}$ is complete. Since a compact metric space is automatically complete, the Hahn metric on a torus is complete.

Hahn [4] established the completeness of $S_{X}$ when $X$ is a proper plane region.

\section{The hahn metric for a torus.}

Let $X$ be a compact Riemann surface of genus one; that is, a torus. We shall show that the Hahn metric for $X$ is real-analytic and has constant curvature zero. This is a consequence of the fact that the Hahn metric is invariant under the group of conformal automorphisms of $X$ as the following theorem shows.

Theorem 3. Let $X$ be a compact Riemann surface of genus one. Suppose that $\rho(z)|d z|$ is a positive metric on $X$ that is invariant under the group $\operatorname{Aut}(X)$. Let $\pi: C \rightarrow X$ be an analytic unversal covering. Then $\pi^{*}(\rho(z)|d z|)=c|d z|$ for some positive constant $c$. In particular, $o(z)|d z|$ is real-analytıc and has constant curvature zero.

Proof. Let $\Gamma$ be the group of cover transformations associated with the covering $\pi: C \rightarrow X$. $\Gamma$ is generated by two translations $T_{1}$ and $T_{2}$, where $T_{\imath}(z)=$ $z+\omega_{\imath}(\imath=1,2), \omega_{1}$ and $\omega_{2}$ are nonzero complex numbers and $\operatorname{Im}\left(\omega_{1} / \omega_{2}\right) \neq 0$. Define $\tilde{\rho}(z)|d z|=\pi^{*}(\rho(z)|d z|)$. We shall show that $\tilde{\rho}(z)$ is constant on $C$ by demonstrating that the metric $\tilde{\rho}(z)|d z|$ is invariant under all translations of $\boldsymbol{C}$. Let $T(z)=$ $z+\omega$ be any translation of $\boldsymbol{C}$. Then $T$ belongs to $N(\Gamma)$, the normalizer of $\Gamma$ in the group Aut $(\boldsymbol{C})$, since all translations commute. Because $T \in N(\Gamma)$, there exists $\phi \in \operatorname{Aut}(X)$ such that $\pi \circ T=\phi \circ \pi[9$, p. 139]. Now,

$$
\begin{aligned}
T^{*}(\tilde{\rho}(z)|d z|) & =T^{*}\left(\pi^{*}(\rho(z)|d z|)\right)=(\pi \circ T)^{*}(\rho(z)|d z|) \\
& =\pi^{*}\left(\phi^{*}(\rho(z)|d z|)\right)=\pi^{*}(\rho(z)|d z|) \\
& =\tilde{\rho}(z)|d z|
\end{aligned}
$$

or 


$$
\tilde{\rho}(z)=\tilde{\rho}(T(z))\left|T^{\prime}(z)\right|=\tilde{\rho}(z+\omega) .
$$

Because $\omega$ is an arbitrary complex number, we conclude that $\tilde{\rho}$ is constant, say $\tilde{\rho}=c$. Thus, $\tilde{\rho}(z)|d z|=c|d z|$ is just a scalar multiple of the euclidean metric which is real-analytic and has constant curvature zero. Since the analytic function $\pi$ is locally injective and $\pi^{*}(\rho(z)|d z|)=c|d z|$, we may conclude that $\rho(z)|d z|$ is real-analytic and has constant curvature zero.

Note that the constant $c$ in Theorem 3 probably depends on the torus $X$. It would be interesting to determine the value of $c$, say in terms of $\omega_{1}$ and $\omega_{2}$.

Since exp: $\boldsymbol{C} \rightarrow \boldsymbol{C} \backslash\{0\}$ is a universal covering, it is possible to establish the analog of Theorem 3 for $C \backslash\{0\}$ in place of a torus. In fact, we could show that

$$
\exp ^{*}\left(S_{C \backslash 00}(z)|d z|\right)=c|d z|,
$$

or equivalently,

$$
S_{C \backslash 01}(z)|d z|=\frac{c|d z|}{|z|}
$$

for some positive constant $c$. In the next section we explicitly determine $S_{C \backslash 0\}}(z)|d z|$ by a different method.

\section{The Hahn metric on doubly connected Riemann surfaces.}

By a doubly connected Riemann surface we understand a surface with fundamental group isomorphic to $Z$. Any doubly connected Riemann surface is conformally equivalent to exactly one of the following plane regions: (i) $\boldsymbol{C} \backslash\{0\}$, (ii) $\boldsymbol{B} \backslash\{0\}$, or (iii) an annulus $A_{R}=\{z: 1 / R<|z|<R\}$ where $R \in(1, \infty)[3$, p. 192]. Thus, it suffices to determine the Hahn metric for the punctured plane, the punctured disk and an annulus in order to know it for any doubly connected surface. We shall explicitly calculate the Hahn metric and its curvature in each of these three cases.

(i) $\boldsymbol{C} \backslash\{0\}$. We will prove that

$$
S_{C \backslash\{0\}}(z)|d z|=\frac{|d z|}{4|z|},
$$

so that the Hahn metric for the punctured plane is just one quarter of the logarithmic metric. It follows that the curvature is identically zero. Let us establish (1). Suppose $f \in \mathcal{S}(\boldsymbol{B}, \boldsymbol{C} \backslash\{0\})$ and $f(0)=a$. The Koebe 1/4-theorem applied to $(f-a) / f^{\prime}(0)$ gives

$$
\frac{1}{4} \leqq \frac{|a|}{\left|f^{\prime}(0)\right|}
$$

with equality if and only if $f$ is a conformal mapping of $\boldsymbol{B}$ onto $\boldsymbol{C}$ slit along a ray emanating from the origin in the direction opposite $a$ and $f(0)=a$. 
Theorem 4. Let $X$ be a plane region, $X \neq C$, and $\delta_{X}(z)=\operatorname{dist}(z, C \backslash X)$. Then

$$
\frac{1}{4 \delta_{X}(z)} \leqq S_{X}(z) \leqq \frac{1}{\delta_{X}(z)} .
$$

Proof. First, we establish the lower bound which is due to Hahn [4]. Fix $a \in X$ and select $b \in \partial X$ with $|a-b|=\delta_{X}(a)$. Since $X \subset C \backslash\{b\}$, the monotonicity property of the Hahn metric yields

$$
S_{X}(a) \geqq S_{C \backslash b\}}(a)=\frac{1}{4|a-b|}=\frac{1}{4 \delta_{X}(a)} .
$$

The upper bound is also elementary to establish. Define $f(z)=a+\delta_{X}(a) z$. Then $f \in \mathcal{S}(\boldsymbol{B}, X), f(0)=a$ and $f^{\prime}(0)=\delta_{X}(a)$ so that

$$
S_{X}(a) \leqq \frac{1}{\left|f^{\prime}(0)\right|}=\frac{1}{\delta_{X}(a)} .
$$

(ii) $\boldsymbol{B} \backslash\{0\}$. We shall demonstrate that

$$
S_{B \backslash 0 !}(z)|d z|=\frac{(1+|z|)|d z|}{4|z|(1-|z|)} .
$$

Since $S_{B \backslash 0\}}(z)|d z|$ is invariant under the group $\operatorname{Aut}(\boldsymbol{B} \backslash\{0\})$ which consists of all rotations about the origin, we obtain $S_{\boldsymbol{B} \backslash 01}(z)=S_{\boldsymbol{B} \backslash 0\}}(|z|)$ for $z \in \boldsymbol{B} \backslash\{0\}$. Thus, we need only establish (2) for $z=a \in(0,1)$. We want to determine $f \in \mathcal{S}(\boldsymbol{B}, \boldsymbol{B} \backslash\{0\})$ that satisfies $f(0)=a$ and maximizes $\left|f^{\prime}(0)\right|$. Let $\Omega=f(\boldsymbol{B})$. Circular symmetrize $\Omega$ with respect to the positive real axis. Since $\Omega$ is simply connected and omits 0 , the circular symmetrization of $\Omega$ is contained in the simply connected region $\Omega^{*}=\boldsymbol{B} \backslash(-1,0]$. Then $\left|f^{\prime}(0)\right| \leqq\left|\phi^{\prime}(0)\right|$, where $\phi: B \rightarrow \Omega *$ is a conformal mapping that satisfies $\phi(0)=a[5$, p. 84$]$. Thus,

$$
S_{B \backslash(0)}(a)=\frac{1}{\left|\phi^{\prime}(0)\right|} .
$$

The elementary conformal mapping $\phi$ can be determined in a straightforward manner; direct calculation yields (2).

Next, we calculate the curvature of $S_{B \backslash(0)}(z)|d z|$. By definition the curvature is

$$
\kappa(z)=-\frac{\Delta \log S_{B \backslash 01}(z)}{S_{B \backslash\{0\}}^{2}(z)} .
$$

It is advantageous to change to polar coordinates. Let $u(r)=\log S_{B \backslash 01}(r)$. Then $u$ is independent of $\theta$ so

$$
\Delta u=u_{r r}+\frac{1}{r} u_{r}
$$

We obtain

$$
-4<\kappa(z)=-\frac{32|z|\left(1+|z|^{2}\right)}{(1+|z|)^{4}}<0
$$


Notice that

$$
\begin{aligned}
& \lim _{z \rightarrow 0} \kappa(z)=0, \\
& \lim _{|z| \rightarrow 1} \kappa(z)=-4 .
\end{aligned}
$$

(iii) $A_{R}$. Let $S_{R}(z)|d z|$ denote the Hahn metric for $A_{R}$. We shall express $S_{R}(z)$ explicitly in terms of Jacobian elliptic functions. Since $S_{R}(z)|d z|$ is invariant under $\operatorname{Aut}\left(A_{R}\right)$ which contains all rotations about the origin, we conclude that $S_{R}(z)=S_{R}(|z|)$ for $z \in A_{R}$. Consequently, it suffices to determine $S_{R}(a)$ for $a \in(1 / R, R)$. This is equivalent to determining $f \in \mathcal{S}\left(\boldsymbol{B}, A_{R}\right)$ that satisfies $f(0)=a$ and maximizes $\left|f^{\prime}(0)\right|$ over the class of all such functions. Assume $f \in \mathcal{S}\left(\boldsymbol{B}, A_{R}\right)$, $f(0)=a$ and $f(\boldsymbol{B})=\Omega$. The circular symmetrization of $\Omega$ with respect to the positive real axis is contained in the simply connected region $\Omega^{*}=A_{R} \backslash(-R,-1 / R)$. Therefore, if $\phi: B \rightarrow \Omega^{*}$ is a conformal mapping with $\phi(0)=a$, then $\left|f^{\prime}(0)\right| \leqq$ $\left|\phi^{\prime}(0)\right|[5$, p. 84]. This yields

$$
S_{R}(a)=-\frac{1}{\left|\phi^{\prime}(0)\right|}=\left|\Psi^{\prime}(a)\right|,
$$

where $\Psi=\phi^{-1}$.

Now, we want to explicitly determine $\Psi$. Let $s n, c n$ and $d n$ denote the Jacobian elliptic functions relative to the parameter $\tau$. The basic facts that we require about these functions are contained in [11, Chapter VI, Section 3]. Let $k=\sqrt{\lambda(\tau)}$, where $\lambda$ denotes the elliptic modular function that is normalized by $\lambda(0)=1, \lambda(1)=\infty, \lambda(\infty)=0$. If

$$
\begin{aligned}
& K=K(k)=\int_{0}^{1} \frac{d t}{\sqrt{\left(1-t^{2}\right)\left(1-k^{2} t^{2}\right)}}, \\
& K^{\prime}=K^{\prime}(k)=K\left(\sqrt{1-\bar{k}^{2}}\right),
\end{aligned}
$$

then for $\tau=\imath K^{\prime} / K$ the function

$$
f(z)=\sqrt{\frac{1-c n(\bar{z})}{1+c n(z)}}
$$

maps the rectangle $R\left(K, K^{\prime}\right)=\left\{z:|\operatorname{Re}(z)|<K,|\operatorname{Im}(z)|<K^{\prime}\right\}$ conformally onto the unit disk $\boldsymbol{B}$ with $f(0)=0$ [11, p. 297]. The function $f$ is symmetric about the real axis. By making use of elementary identities for the Jacobian elliptic functions, we find that

$$
f^{\prime}(z)=\frac{d n(z)}{1+\operatorname{cn}(z)} .
$$

In particular, $f^{\prime}(0)=1 / 2$ since $c n(0)=1=d n(0)$. The function $L(z)=(K \log z) /$ $\log R$ maps $\Omega^{*}$ conformally onto $R\left(K, K^{\prime}\right)$; here we select $\tau$ so that $K^{\prime} / K=\pi /$ $\log R$. Then $f \circ L$ is a conformal mapping of $\Omega^{*}$ onto $\boldsymbol{B}$. Set $b=f(L(a)) \in(-1,1)$ and define $T(z)=(z-b) /(1-b z)$. The function $T$ is a conformal automorphism of $\boldsymbol{B}$ that sends $b$ to the origin. Finally, $\Psi=T \circ f \circ L$ is a conformal mapping of 
$\Omega^{*}$ onto $\boldsymbol{B}$ with $\Psi(a)=0$. Now,

Thus,

$$
\begin{aligned}
\Psi^{\prime}(a) & =\frac{K}{a \log R} \frac{f^{\prime}\left(\frac{K \log a}{\log R}\right)}{1-f^{2}\left(\frac{K \log a}{\log R}\right)} \\
& =\frac{K}{2 a \log R} \cdot \frac{d n\left(\frac{K \log a}{\log R}\right)}{\operatorname{cn}\left(\frac{K \log a}{\log R}\right)} .
\end{aligned}
$$

$$
S_{R}(z)=\frac{K}{2|z| \log R} \cdot \frac{\operatorname{dn}\left(\frac{K \log |z|}{\log R}\right)}{\operatorname{cn}\left(\frac{K \log |z|}{\log R}\right)} .
$$

It is possible to express the constant $K$ directly in terms of $R$. From [2, pp. $385,410]$ we have

$$
\begin{aligned}
K & =\frac{\pi}{2} \prod_{n=1}^{\infty}\left(1-q^{2 n}\right)^{2}\left(1+q^{2 n-1}\right)^{4} \\
& =\frac{\pi}{2}\left(1+2 \sum_{n=1}^{\infty} q^{n^{2}}\right)^{2}
\end{aligned}
$$

where $q=\exp (\imath \pi \tau)$. Because $\tau=i K^{\prime} / K=i \pi / \log R$ we obtain

$$
K=\frac{\pi}{2}\left[1+2 \sum_{n=1}^{\infty} \exp \left(-\frac{n^{2} \pi^{2}}{\log R}\right)\right]^{2}
$$

For purposes of comparison we give the hyperbolic metric for $A_{R}$; it is

$$
\left.\lambda_{R}(z)|d z|=\frac{\pi}{4|z| \log R}-\frac{1}{\cos \left(\frac{\pi}{2} \log |z|\right.} \log R\right) .
$$

There is a curious connection between the Hahn metric and the hyperbolic metric. For $\tau=\imath \infty 0$ we have $k=0$. In this case, $\operatorname{sn}(z)=\sin (z), c n(z)=\cos (z)$, $d n(z)=1$ and $K(0)=\pi / 2[10$, p. 216]. Thus, for $\tau=\imath \infty$ the Hahn metric becomes the hyperbolic metric.

We are interested in the curvature of $S_{R}(z)|d z|$. The calculations are simplified if we use the identity

$$
\frac{d n(u)}{c n(u)}=k \operatorname{sn}\left(u+K+\imath K^{\prime}\right)
$$

[2, p. 388] and change to polar coordinates. Straightforward calculation give

$$
-4<\kappa(z)=-4+4 k^{2} \frac{c n^{4}\left(\frac{K \log |z|}{\log R}\right)}{d n^{4}\left(\frac{K \log |z|}{\log R}\right)}<0 .
$$


Observe that

$$
\lim _{z \rightarrow \partial A_{R}} \kappa(z)=-4
$$

\section{Curvature properties of the Hahn metric.}

Our final goal is to investigate the curvature of the Hahn metric. We start by recalling the curvature in some particular cases. Suppose $X$ is a simply connected Riemann surface; then $X$ is conformally equivalent to exactly one of $\boldsymbol{B}, \boldsymbol{C}$ and $\boldsymbol{P}$. If $X$ is conformally equivalent to either $\boldsymbol{C}$ or $\boldsymbol{P}$, then the Hahn metric is trivial. When $X$ is conformally equivalent to $\boldsymbol{B}$, the Hahn metric coincides with the hyperbolic metric. In this situation it has constant curvature -4. If $X$ is conformally equivalent to the punctured plane $C \backslash\{0\}$ or torus, then $S_{X}(z)|d z|$ has constant curvature zero. Finally, if $X$ is conformally equivalent to a the punctured disk $\boldsymbol{B} \backslash\{0\}$ or an annulus, then the curvature lies strictly between -4 and 0 . For a general Riemann surface $X \neq \boldsymbol{C}, \boldsymbol{P}$ it is only known that $S_{X}(z)|d z|$ is a continuous metric [4]. It is an open question whether it is of class $C^{2}$ in the general case. Thus, it does not make sense to speak of the curvature of the Hahn metric of a general Riemann surface. For this reason we introduce generalized notions of curvature which were motivated by Heins' definition of $S K$ metrics [6].

Definition. Let $\rho(z)|d z|$ be a continuous metric on a Riemann surface. Suppose $p \in X$ and $z$ is any local coordinate in a neighborhood of $p$ with $z(p)=0$. Define

$$
\begin{gathered}
\bar{\kappa}(p)=-\frac{4 \lim _{r \rightarrow 0} \inf \frac{1}{r^{2}}\left[\frac{1}{2 \pi} \int_{0}^{2 \pi} \log \rho\left(r e^{2 \theta}\right) d \theta-\log \rho(0)\right]}{\rho^{2}(0)} \\
\underline{\kappa}(p)=-4 \lim _{r \rightarrow 0} \sup \frac{1}{r^{2}}\left[\frac{1}{2 \pi} \int_{0}^{2 \pi} \log \rho\left(r e^{\imath \theta}\right) d 0-\log \rho(0)\right] \\
\rho^{2}(0)
\end{gathered} .
$$

$\underline{\kappa}(p)(\bar{\kappa}(p))$ is called the generalized upper (lower) curvature of $\rho(z)|d z|$ at $p$.

Observe that if $\rho(z)|d z|$ is of class $C^{2}$ in a neighborhood of $p$, then

$$
\Delta \log \rho(0)=4 \lim _{r \rightarrow 0} \frac{1}{r^{2}}\left[\frac{1}{2 \pi} \int_{0}^{2 \pi} \log \rho\left(r e^{2 \theta}\right) d \theta-\log \rho(0)\right] .
$$

In this situation

$$
\underline{\kappa}(p)=\kappa(p)=\bar{\kappa}(p),
$$

where $\kappa(p)$ denotes the Gaussian curvature of $\rho(z)|d z|$ at $p$. In general, the numerator of the expression in $(3) ;((4))$ is a generalized lower (upper) Laplacian for $\log \rho$.

We shall prove that the Hahn metric has generalized upper curvature at 
least -4 . The method that we employ is closely related to the work of Suita [13].

LEMMA. Suppose that $\sigma(z)|d z|$ is a positive metric of class $C^{2}$ in a neighborhood of a point $a \in C$. Suppose that $\rho(z)|d z|$ is a contrnuous metric in $a$ neighborhood of $a, \rho(a)=\sigma(a)$ and $\rho(z) \leqq \sigma(z)$ in a nerghborhood of $a$. Then $\kappa(a, \sigma)$ $\leqq \underline{\kappa}(a, \rho)$.

Proof. Clearly, $\log (\sigma(z) / \rho(z))$ is continuous and nonnegative in a neighborhood of $a$ and has a local minimum at $a$. It follows that

Hence,

$$
\frac{1}{2 \pi} \int_{0}^{2 \pi} \log \frac{\sigma}{\rho}\left(a+r e^{2 \theta}\right) d \theta \geqq 0=\log \frac{\sigma(a)}{\rho(a)} .
$$

$$
\begin{aligned}
& \frac{1}{r^{2}}\left[\frac{1}{2 \pi} \int_{0}^{2 \pi} \log \sigma\left(a+r e^{i \theta}\right) d \theta-\log \sigma(a)\right] \\
& \geqq \frac{1}{r^{2}}\left[\frac{1}{2 \pi} \int_{0}^{2 \pi} \log \rho\left(a+r e^{i \theta}\right) d \theta-\log \rho(a)\right]
\end{aligned}
$$

for all $r>0$ sufficiently small. If we take the limit superior of both sides of this inequality as $r \rightarrow 0$, then we obtain the desired result since $\sigma$ is of class $C^{2}$ in a neighborhood of $a$.

THEOREm 5. Suppose $X$ is a Riemann surface that is not conformally equivalent to either $\boldsymbol{C}$ or $\boldsymbol{P}$. Then the Hahn metruc $S_{X}(z)|d z|$ has generalized upper curvature at least -4 at each point of $X$.

Proof. We assume that $X$ is actually a region in $C$ and fix $p \in X$. If $X$ is actually a Riemann surface, then we perform similar calculations in terms of any local coordinate at $p$. Let $\phi \in \mathcal{S}(\boldsymbol{B}, X)$ be an extremal function for $S_{X}(p)$; that is, $\phi(0)=p$ and $S_{X}(p)=1 /\left|\phi^{\prime}(0)\right|$. Thus,

$$
S_{X}(\phi(0))\left|\phi^{\prime}(0)\right|=1=\lambda_{B}(0),
$$

where $\lambda_{B}(z)|d z|$ is the hyperbolic metric on $\boldsymbol{B}$. For any $a \in \boldsymbol{B}$ define

$$
\phi_{a}(z)=\phi\left(\frac{z+a}{1+\bar{a} z}\right)
$$

Then $\dot{\phi}_{a} \in \mathcal{S}(\boldsymbol{B}, X)$ and $\phi_{a}(0)=\phi(a)$ so

or

$$
S_{X}(\phi(a)) \leqq \frac{1}{\left|\phi_{a}^{\prime}(0)\right|}=\frac{1}{\left|\phi^{\prime}(a)\right|\left(1-|a|^{2}\right)}
$$

$$
S_{X}(\phi(a))\left|\phi^{\prime}(a)\right| \leqq \frac{1}{1-|a|^{2}}=\lambda_{B}(a)
$$

This implies that

$$
\phi^{*}\left(S_{X}(z)|d z|\right) \leqq \lambda_{B}(z)|d z|
$$

with equality at the origin. Let $\Psi=\phi^{-1}$. Then 


$$
S_{X}(z)|d z| \leqq \Psi^{*}\left(\lambda_{B}(z)|d z|\right)
$$

on $\phi(\boldsymbol{B})$ with equality at $p$. Because curvature is a conformal invariant, $\Psi^{*}\left(\lambda_{B}(z)|d z|\right)$ has constant curvature -4 on $\phi(\boldsymbol{B})$. The theorem now follows immediately from the preceding lemma.

If $X$ is any Riemann surface such that $S_{X}(z)|d z|$ is of class $C^{2}$, then Theorem 5 actually implies that $S_{X}(z)|d z|$ has Guassian curvature at least -4 . In all of our examples, the curvature of the Hahn metric was either identically zero or else strictly negative. Is it true that for an arbitrary Riemann surface $X \neq \boldsymbol{C}, \boldsymbol{P}$, the generalized lower curvature of the Hahn metric is nonpositive? Also, in our examples, the curvature of the Hahn metric approached -4 at any point of an analytic boundary contour of $X$. Is this true in more generality?

$A D D E D$ IN PROOF. Recently, the author discovered that the Hahn metric was also considered in the following papers. $145-168$.

N. Mok, The Serre problem on Riemann surfaces, Math. Ann. 258 (1981),

Y.-T. Siu, All plane regions are Banach-Stein, Manuscripta Math. 14 (1974), 101-105.

\section{REFERENCES}

[1] C. Carathéodory, Theory of Functions, Vol. II, 2nd ed., Chelsea Publ. Co., New York, 1960.

[2] E.T. Copson, An Introduction to the Theory of Functions of a Complex Variable, Oxford Univ. Press, London, 1935.

[3] H.M. FARKAS AND I. KRA, Riemann Surfaces, Graduate Texts in Mathematics, vol. 71, Springer-Verlag, New York, 1980.

[4] K. T. HaHn, Some remarks on a new pseudo-differential metric, Ann. Polon. Math. 39 (1981), 71-81.

[5] W.K. HAYman, Multivalent Functions, Cambridge Tracts in Mathematics and Mathematical Physics, no. 48, Cambridge Univ. Press, 1967.

[6] M. Heins, On a class of conformal metrics, Nagoya Math. J. 21 (1962), 1-60.

[7] J.A. Jenkins, On the existence of certain general extremal metrics, Ann. of Math. 66 (1957), 440-453.

[8] J.A. JENKINS AND N. Suita, On the representation and compactification of Riemann surfaces, Bull. Inst. Math. Acad. Sinica 6 (1978), 423-427.

[9] J. Lehner, A Short Course in Automorphic Functions, Holt, Rinehart and Winston, New York, 1966.

[19] G. Moretti, Functions of a Complex Variable, Prentice-Hall, Englewood Cliffs, New Jersey, 1964.

[11] Z. Nehari, Conformal Mapping, McGraw-Hill, New York, 1952.

[12] K. Strebel, On quadratic differentials with closed trajectories and second order poles, J. Analyse Math. 19 (1967), 373-382.

[13] N. Suita, On a metric induced by analytıc capacıty, Kōdaı Math. Sem. Rep. 25 (1973), 215-218.

Department of Mathematical Sciences

UNIVERSITY OF CINCINNATI

CinCINNATi, Oino 45221

U.S.A. 